\title{
A common misunderstanding in lung ultrasound: the comet tail artefact
}

\author{
Francis Chun Yue Lee ${ }^{1-3}$, Christian Jenssen ${ }^{4}$, Christoph F Dietrich ${ }^{5,6}$
}

\begin{abstract}
${ }^{1}$ Acute and Emergency Care Centre, Khoo Teck Puat Hospital, Singapore, ${ }^{2}$ Yong Loo Lin School of Medicine, National University of Singapore, ${ }^{3}$ Lee Kong Chian School of Medicine, National Technological University, Singapore, ${ }^{4}$ Departmentof Internal Medicine, Krankenhaus Märkisch Oderland, Strausberg/Wriezen, Germany, ${ }^{5}$ Caritas Krankenhaus Bad Mergentheim, Germany, ${ }^{6}$ Ultrasound Department, The First Affiliated Hospital of Zhengzhou University, Zhengzhou, China
\end{abstract}

\begin{abstract}
The comet tail artefact is probably one of the most commonly and imprecisely used to describe vertical artefacts found in lung ultrasound. Two distinct artefacts are commonly observed: the lung comets and the B-lines. Both artefacts differ with regard to generation mechanism and clinical significance. This review explores the current understanding and use of these two artefacts in lung ultrasound and suggests how to avoid the pitfalls related to confusing comet tail artefacts with other vertical artefacts.
\end{abstract}

Keywords: B-lines; comet tail artifact; lung comets; ring-downlung; ultrasound

Traditionally, ultrasound (US) was deemed unsuitable for the study of the lung because of the acoustic properties of air. Human lungs contain more than $99 \%$ air [1] and air has high acoustic impedance to US. The acoustic mismatch between the lung air and the adjacent extrapulmonary tissue creates an US image of disorganized air artefacts without any discernible anatomical features. However, it is now recognized that distinct artefacts and the way they change in disease processes could be used in making US diagnosis and guiding treatment.

Of the many artefacts described in the lung ultrasound (LUS) literature [2-8], the most often talked about and perhaps creating the most confusion amongst practitioners is the comet tail artefact (CTA). CTA is so named primarily due to their appearance on US but it now known that many disparate linear vertical artefacts share this morphology. In the present short review, starting with the

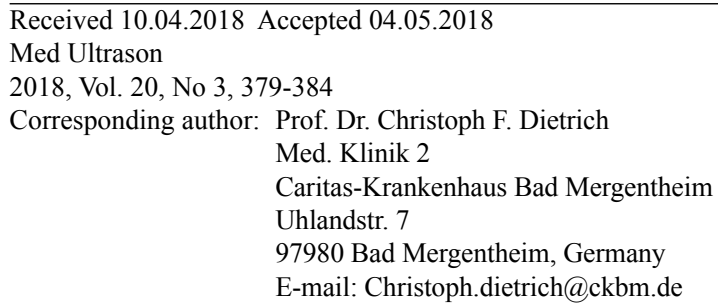

CTA, we explore the genesis of the various vertical lung artefacts, their differentiation and their impact for clinical decision-making.

\section{The comet tail artefact (CTA)}

Ziskin et al first characterized the CTA in a patient with an abdominal gunshot wound [9]. They described the CTA as a dense tapering trail of echoes just distal to a strongly reflective surface and proposed a reverberation mechanism for its genesis. In a follow-up paper [10], Thickman et al. presented several clinical situations where CTAs could be seen. They observed that CTA can be generated when there is an acoustic mismatch at a sonographic interface, most notably an air-tissue interface; others include interfaces presented by bones, stones and foreign bodies. A distinct "CTA" that is continuous and increasing in intensity with depth was also mentioned and the authors alluded to the fact that these were commonly referred to as ring-down artefacts (RDA).

\section{The ring down artefact $(\mathrm{RDA})$}

Arvuch et al demonstrated in experiments that emitted waves from the resonant vibrations within a 
bubble tetrahedral complex (four air bubbles arranged in a tetrahedron formation and trapping a bugle-shaped column of fluid within), in response to insonating US, creates the RDA [11]. Morphologically, the RDA is a linear artefact in the axis of the insonated ultrasound wave that starts from the point of origin and "ringsdown" to the end of the screen without fading. In contrast, a CTA, which is created via a reverberation mechanism dependent on acoustic impedance differences between mediums, fades and tapers with increasing depth.

Depending on the gain setting on the US, the RDA may appear as continuous or as successive stacks of short horizontal bands. The thickness of the horizontal bands and the distance between them (periodicity) is always constant, unlike in CTA (fig 1).

\section{The misunderstanding in lung ultrasound}

Despite the superficial resemblance, CTA and RDA differ in respect of genesis, morphology and differing clinical significance in LUS [12]. It is perhaps the imprecise use of the term CTA in lung US (LUS) that has led to misunderstandings, manifesting in daily practice and published literature. Lichtenstein et al introduced the "CTA" as a sign of interstitial syndrome [13]. In their initial paper, they made a distinction between the long and short type of CTA and clearly stated that only the long type was valid for interstitial syndrome diagnosis. They presented images and described this long type CTA as a hyperechoic vertical artefact that spreads like a laser ray to the end of the screen; a description that clearly matches the RDA. However, in their ensuing discussion, they continued to use the term CTA and in so doing ascribed a reverberation mechanism to the RDA they were talking about.

When Lichtenstein later developed the alphabetic nomenclature of LUS artefacts [14], the term B-lines was assigned to the RDA (which he called CTA) used in interstitial syndrome diagnosis. However, the term CTA became stuck in LUS literature and is used interchangeably with B-lines. This has caused varying interpretations and confusion in LUS:

- The term "CTA" used but images showed B-lines (numerous examples)

- The term "CTA" used but images showed other type of vertical artefacts $[15,16]$.

Several authors who were aware of this problem have emphasized the importance of making a distinction [2,17-19] and international evidence-based recommendations for point-of-care lung ultrasound recommended to avoid the term CTA to describe B-lines [20].

\section{B-lines: a ring-down artefact}

B-lines is a specific term for RDA found in LUS and they must have the following characteristics, in addition to the standard properties of RDA:

- it originates at the pleural line (PL),

- it has a strong light ray-like appearance, obliterating other background LUS artefacts,

- it moves with lung sliding.

Physiologic B-lines

The normal lung is characterized by the absence or presence of very few B-lines; less than three per field of view. These "physiologic" B-lines, seen in only $10 \%$ of young healthy subjects [21], are often a transient phenomenon and change with posture. The dependence of B-lines creation on availability of a medium that could create a resonating vibration (bubble-tetrahderal complexes or equivalent) probably explains why they are more likely found in the better perfused dependent areas of the lung or at the thicker interlobular septa [5,15]. Age related changes in the lung parenchyma such as fibrosis and sub-pleural lesions account for increased B-lines observed in $37 \%$ of the elderly [21].

\section{Pathologic B-lines}

In pathological processes involving the lung parenchyma, fluid, inflammatory infiltrates or cellular content progressively increase, greatly enhancing the environment for the generation of B-lines (fig 2). As a guide, a finding of a cluster of three or more B-lines per intercostal space may be pathological. However, the number of B-lines and the number of chest areas positive for (multiple) B-lines increases with age [21-23] and therefore one must be cautious of a false positive interpretation in older persons. The often-used term "interstitial syndrome" $[13,24]$ is a description of ultrasound findings of pathological B-lines. The term is not specific or synonymous to acute interstitial lung disease $[6,25]$.

These are examples of conditions that produce a generalized and often bilateral interstitial syndrome: cardiogenic pulmonary edema [13], acute or chronic interstitial lung disease $[26,27]$ or acute lung injury / acute respiratory distress syndrome [28].

Focal interstitial syndrome may be observed in relation to pneumonia [13,29], pulmonary contusion [30,31], lung tumors $[29,32]$ or other pulmonary consolidating processes [29,33].

As B-lines are sensitive but non-specific for pathological lung parenchyma changes, the findings of interstitial syndrome must be correlated with the following information [34] to make a clinical diagnosis:

- distribution of B-lines (interstitial syndrome) in the lung fields: patchy, uniform, symmetry, 

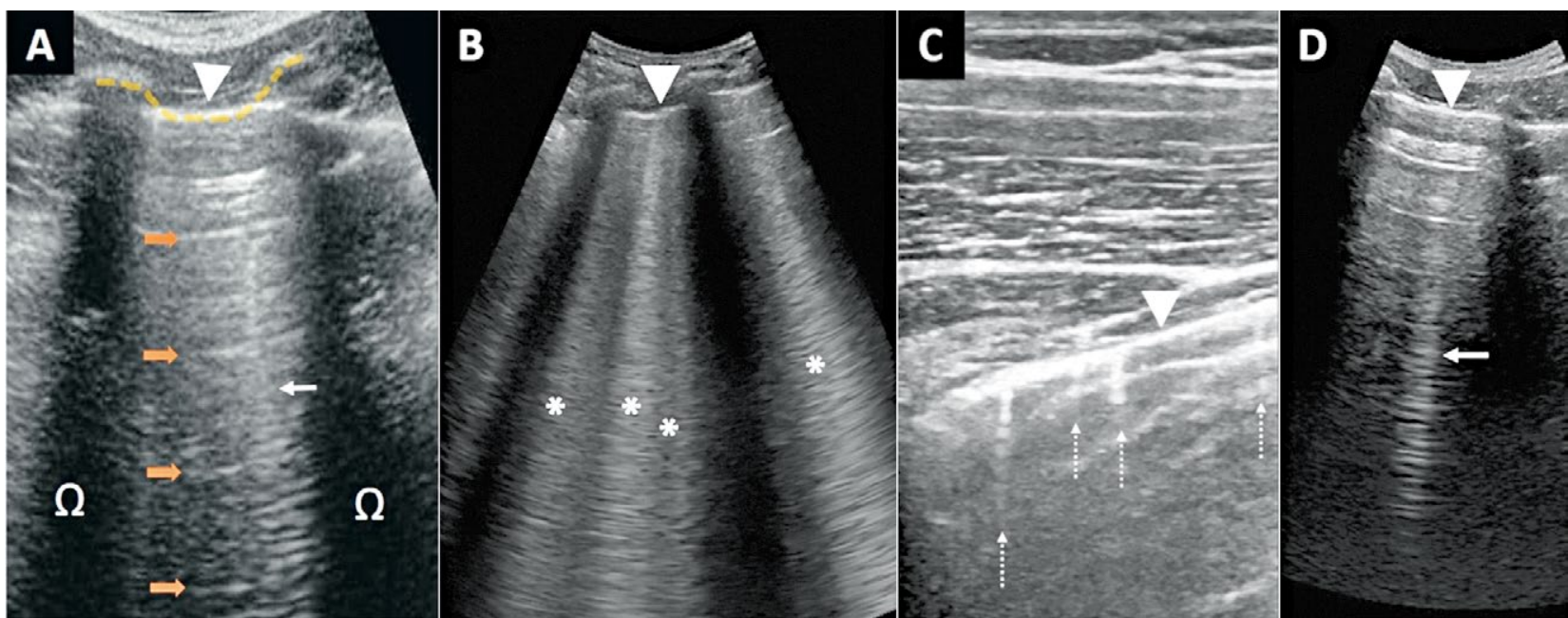

Fig 1. A comparison of the common vertical artefacts. (A) The normal LUS appearance. The (pleural line) PL (arrow head) is at a level just below the two adjacent ribs and together trace the bat sign (yellow dotted line). The ribs cast acoustic shadows $(\Omega)$, forming the boundaries of the sonographic lung field (SLF), the area of LUS study. Within the SLF are equally spaced repetition artefacts called A-lines (orange arrows). An incidental Z-line (arrow) is seen;(B) B-lines (*) are RDA that start at the PL and radiate to the end of the screen without fading. Note that the B-lines are made up of closely stacked horizontal artefacts that give an almost continuous appearance; (C) LUS of a normal lung showing short reverberation artefacts known as lung comets (dotted arrows). They start from the PL (arrowhead) and move with lung sliding. Their appearance is obviously different from B-lines; (D) Another type of reverberation artefact commonly seen in LUS, the Z-line (arrow) is a weak vertical artefact that appears to blend with other background artefacts. Note the lack of relationship with the PL (arrowhead). It does not move with lung sliding. They are often misinterpreted as B-lines by inexperienced clinicians.

- evaluation of PL morphology [35,36]: lung sliding, thickening, unevenness,

- other LUS features: consolidation, sub-pleural lesions, effusion,

- clinical information of the patient: oxygen saturation, blood gases, other laboratory findings,

- medical history of the patient: e.g. history of pleuritis, thorax injury or surgery, interstitial lung disease or connective-tissue/ rheumatic disease.

In the clinical context of acute dyspnea and desaturation, the utility of B-lines in LUS diagnosis is limited in patients with pre-existing interstitial syndrome unless prior LUS reports and images are available for comparison. Additional US studies such as echocardiography are often helpful in clarifying the cause of the interstitial syndrome.

\section{What kinds of CTA can be seen in LUS?}

\section{Lung comets}

In the normal lung, a specific type of CTA can be seen. These are short (almost always less than $1 \mathrm{~cm}$ ) vertical artefacts that taper and fade with increasing depth. Similar to B-lines, they arise from the PL and move with lung sliding; these signs point to their origin from a reverberation mechanism occurring at the peripheral lung parenchyma or the inter-pleural layer and the dependence on the apposition of the visceral and parietal
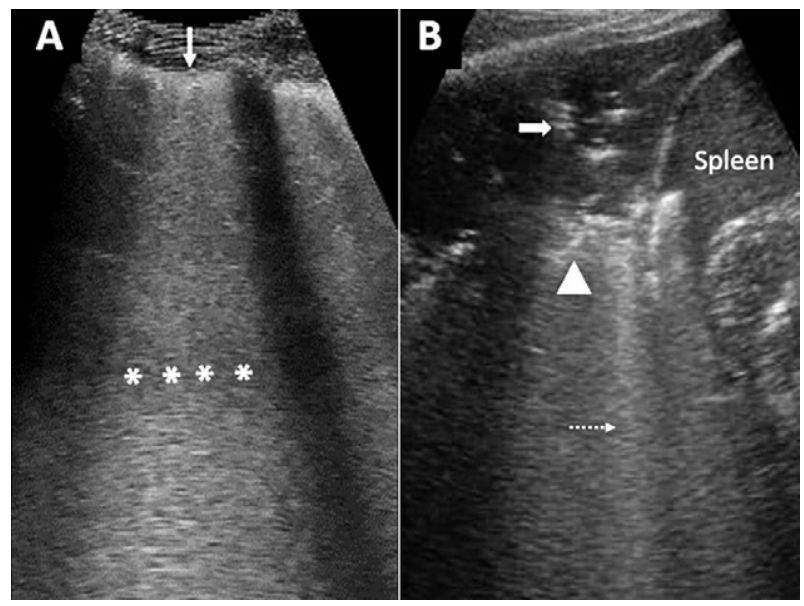

Fig 2. Demonstration of RDA in various conditions: (A) LUS image of a patient with acute respiratory distress syndrome. Extensive number of B-lines (*) are present, blending into a white sheet, obscuring all other background LUS artefacts. Note the thickened and uneven pleural which points to an inflammatory etiology in this case;(B) A consolidation at the costophrenic recess causing the loss of curtain sign [42], exposing the lateral diaphragm and allowing the visualization of the spleen beneath. The hypoechoic region of the consolidation contains air bronchograms (arrow). The boundary between the consolidated area and the remaining aerated part of the lung is uneven, a feature known as the shred line (arrow head). An incidental RDA arises from the shred line. 
pleura for their creation. They are found in all areas of the lung $[3,14,18,19]$, best visualized with higher frequency transducers. It is interesting to note that studies that use low frequency transducers for LUS do not specifically mention them. Numerous CTA seen together sometimes gives the PL a "beads on a string" appearance. As there is currently no specific term to describe this type of CTA, the authors propose the term 'lung comets' for the purpose of discussion henceforth.

\section{Z-Lines}

Z-lines are observed as vertical artefacts in LUS, especially in thin individuals. Z-lines do not originate at the PL and do not move with lung sliding [37,38]. They are typically weak in appearance, blend with surrounding artefacts (e.g. A-lines) and fade with increasing depth. These characteristics and the varying periodicity and thickness of the horizontal bands that comprises the Zline are testimonial to a reverberation mechanism occurring outside the lung. They have no clinical significance, but it is important not to confuse them with B-lines.

Lung comets versus $B$-lines in disease diagnosis

The visualization of lung comets or B-lines in LUS gives proof that the visceral and parietal pleura are in contact. They are therefore important supporting signs, besides lung sliding, in excluding a pneumothorax.

Verification of B-lines is often taught as a method for ruling out pneumothorax. However, this criterion is limited by low sensitivity because B-lines are rarely found in the normal lung $[17,19]$, especially in the upper parts where pneumothorax tends to occur. This fact was clearly expressed in Lichtenstein's validation of Blines (then called "CTA") in ruling out pneumothorax [38], where none of the subjects with normal lungs had B-lines. B-lines are more useful if their presence is already demonstrated prior to the pneumothorax event, e.g. in an intubated patient with pulmonary edema, and comparisons of scans show the subsequent disappearance.

In contrast, the use of lung comets in this context is much more effective simply because of their ubiquitous nature. In the challenging situation of determining the cause of absent lung sliding (e.g. pneumothorax vs non-ventilation in critical care), one should actively look for lung comets and be less reliant on B-lines. This approach suggested by the authors has not been validated in research but it is already well known as evidenced by images of lung comets used in several discussions on pneumothorax diagnosis [39,40].

Due to their common occurrence in the normal lung, lung comets (c.f. B-lines) should not be used to assess for interstitial syndrome and to do so is to make a diagnosis when there is none.

\section{RDA other than B-lines}

\section{$R D A$ at pathological interfaces}

RDA that do not originate from PL are not B-lines. A classic example is seen at the shred line; the interface between a consolidation and the aerated part of the lung [37]. This region has the right conditions for forming bubble tetrahedral complexes and RDA often arise from it. A lung compressed under the weight of a pleural effusion sometimes generates RDA (named sub B-lines by Lichtenstein) from the visceral pleural. In both these scenarios, these RDAs do not necessarily indicate the presence of interstitial syndrome.

\section{I-lines}

I-lines [37] share all the same characteristics as Blines except they are short, about 3-4 cm in length. They are occasionally seen at the sites of the inter-lobar fissures. They could represent a partially visualized B-line, and currently have no clinical significance assigned to them.

\section{Other described vertical artefacts in lung ultrasound \\ E-lines}

Air in the subcutaneous tissues of the chest wall can cause LUS artefacts that simulate the lung. The sonographer may mistake the layer of air as the PL. On closer examination, the pseudo-PL is uneven and varying in thickness; the ribs are not visible and there is no bat sign. Uncommonly, small pockets of air may create RDA or CTA. These vertical artefacts that arise from the subcutaneous layer are collectively called E-lines $[37,41]$

$W$-lines

$\mathrm{W}$-lines [37] is just a variation of E-lines where the point of origins of the vertical artefacts are scattered at different planes.

\section{Conclusion}

The term CTA in LUS has caused confusion simply because it is used to describe any vertical artefact, in particular B-lines, which is an RDA. CTA and RDA are generated by distinct mechanisms; while reverberation due to acoustic impedance difference is the mechanism of generating CTA, RDA are caused by resonant vibration due to bubble tetrahedral complexes or their equivalents. Their appearance in LUS will depend on the state of the lung to provide the right acoustic environment for their generation, in both healthy and diseased patients. Using pneumothorax and interstitial syndrome as examples, we illustrated that the lung comets (CTAs) and B-lines (RDA) have distinct roles in disease diagnosis and the potential pitfalls if they are simply lumped together and called CTAs. 


\section{References}

1. Armstrong JD, Gluck EH, Crapo RO, Jones HA, Hughes JM. Lung tissue volume estimated by simultaneous radiographic and helium dilution methods. Thorax 1982;37:676679.

2. Dietrich CF, Mathis G, Blaivas M, et al. Lung artefacts and their use. Med Ultrason 2016;18:488-499.

3. Dietrich CF, Mathis G, Blaivas M, et al. Lung B-line artefacts and their use. J Thorac Dis 2016;8:1356-1365.

4. Bonhof JA. Ultrasound Artifacts - Part 1. Ultraschall Med 2016;37:140-153.

5. Jenssen C, Tuma J, Moller K, et al. Ultrasound artifacts and their diagnostic significance in internal medicine and gastroenterology - part 2: color and spectral Doppler artifacts. Z Gastroenterol 2016;54:569-578.

6. Tuma J, Jenssen C, Moller K, et al. Ultrasound artifacts and their diagnostic significance in internal medicine and gastroenterology - Part 1: B-mode artifacts. Z Gastroenterol 2016;54:433-450.

7. Dietrich CF, Ignee A, Greis C, Cui XW, Schreiber-Dietrich DG, Hocke M. Artifacts and pitfalls in contrast-enhanced ultrasound of the liver. Ultraschall Med 2014;35:108-125.

8. Dietrich CF, Ignee A, Hocke M, Schreiber-Dietrich D, Greis C. Pitfalls and artefacts using contrast enhanced ultrasound. Z Gastroenterol 2011;49:350-356.

9. Ziskin MC, Thickman DI, Goldenberg NJ, Lapayowker MS, Becker JM. The comet tail artifact. J Ultrasound Med 1982;1:1-7.

10. Avruch L, Cooperberg PL. The ring-down artifact. J Ultrasound Med 1985;4:21-28.

11. Froehlich E, Pauluschke-Froehlich J, Debove I, Vey W, Klinger C, Dietrich CF. Geriatric ultrasound. Z Gastroenterol 2017;55:277-290.

12. Lichtenstein D, Meziere G, Biderman P, Gepner A, Barre O. The comet-tail artifact: An ultrasound sign of alveolar-interstitial syndrome. Am J Respir Crit Care Med 1997;156:1640-1646.

13. Lichtenstein D. General ultrasound in the critically ill Berlin Heidelberg: Springer-Verlag, 2005.

14. Chan SS. Comet tail artifacts in emergency chest ultrasound. Am J Emerg Med 2007;25:724-725.

15. Sperandeo M, Varriale A, Sperandeo G, et al. Characterization of the normal pulmonary surface and pneumonectomy space by reflected ultrasound. J Ultrasound 2011;14:22-27.

16. Louvet A, Bourgeois JM. Lung ring-down artifact as a sign of pulmonary alveolar-interstitial disease. Vet Radiol Ultrasound 2008;49:374-377.

17. Mathis G. Chest Sonography Berlin Heidelberg: SpringerVerlag, 2011.

18. Richards JR, McGahan JP. Focused Assessment with Sonography in Trauma (FAST) in 2017: What Radiologists Can Learn. Radiology 2017;283:30-48.

19. Volpicelli G, Elbarbary M, Blaivas M, et al. International evidence-based recommendations for point-of-care lung ultrasound. Intensive Care Med 2012;38:577-591.
20. Ciccarese F, Chiesa AM, Feletti F, et al. The senile lung as a possible source of pitfalls on chest ultrasonography and computed tomography. Respiration 2015;90:56-62.

21. Rea G, Trovato GM. A Farewell to B-Lines: Ageing and Disappearance of Ultrasound Artifacts as a Diagnostic Tool. Respiration 2015;90:522.

22. Sferrazza Papa GF, Pellegrino GM, Volpicelli G, et al. Lung Ultrasound B Lines: Etiologies and Evolution with Age. Respiration 2017;94:313-314.

23. Volpicelli G, Mussa A, Garofalo G, et al. Bedside lung ultrasound in the assessment of alveolar-interstitial syndrome. Am J Emerg Med 2006;24:689-696.

24. Trovato GM, Rollo VC, Martines GF, Catalano D, Trovato FM, Sperandeo M. Thoracic ultrasound in the differential diagnosis of severe dyspnea: a reappraisal. Int J Cardiol 2013;167:1081-1083.

25. Hasan AA, Makhlouf HA. B-lines: Transthoracic chest ultrasound signs useful in assessment of interstitial lung diseases. Ann Thorac Med 2014;9:99-103.

26. Targhetta R, Chavagneux R, Balmes P, et al. Sonographic lung surface evaluation in pulmonary sarcoidosis: preliminary results. J Ultrasound Med 1994;13:381-388.

27. Copetti R, Soldati G, Copetti P. Chest sonography: a useful tool to differentiate acute cardiogenic pulmonary edema from acute respiratory distress syndrome. Cardiovasc U1trasound 2008;6:16.

28. Volpicelli G, Caramello V, Cardinale L, Cravino M. Diagnosis of radio-occult pulmonary conditions by realtime chest ultrasonography in patients with pleuritic pain. Ultrasound Med. Biol 2008;34:1717-1723.

29. Wustner A, Gehmacher O, Hammerle S, Schenkenbach C, Hafele H, Mathis G. Ultrasound diagnosis in blunt thoracic trauma. Ultraschall Med 2005;26:285-290.

30. Soldati S, Testa A, Silva FR, Carbone L, Portale G, Silveri NG. Chest ultrasonography in lung contusion. Chest 2006;130:533-538.

31. Volpicelli G, Caramello V, Cardinale L, Mussa A, Bar F, Frascisco MF. Detection of sonographic B-lines in patients with normal lung or radiographic alveolar consolidation. Med Sci Monit 2008;14:CR122-CR128.

32. Acosta CM, Maidana GA, Jacovitti D, et al. Accuracy of transthoracic lung ultrasound for diagnosing anesthesia-induced atelectasis in children. Anesthesiology 2014;120:1370-1379.

33. Lee FC. Lung ultrasound-a primary survey of the acutely dyspneic patient. J Intensive Care 2016;4:57.

34. Singh AK, Mayo PH, Koenig S, Talwar A, Narasimhan M. The Use of M-Mode Ultrasonography to Differentiate the Causes of B Lines. Chest 2018;153:689-696.

35. Li H, Li YD, Zhu WW, et al. High-Resolution Transthoracic Ultrasonography for Assessment of Pleural Lines in Patients With Dyspnea With CT Comparison: An Observational Study. J Ultrasound Med 2017;36:707-716.

36. Lichtenstein D. Lung ultrasound in the critically ill: The BLUE protocol. Cham Switzerland: Springer, 2016.

37. Lichtenstein D, Meziere G, Biderman P, Gepner A. The comet-tail artifact: an ultrasound sign ruling out pneumothorax. Intensive Care Med 1999;25:383-388. 
38. Noble VE. Think ultrasound when evaluating for pneumothorax. J Ultrasound Med 2012;31:501-504.

39. Butts C. The speed of sound: Comet tails and lung sliding: Evaluating for pneumothorax. Emergency Medicine News 2013;35:20.
40. Lichtenstein DA, Meziere G, Lascols N, et al. Ultrasound diagnosis of occult pneumothorax. Crit Care Med 2005;33:1231-1238.

41. Lee FC The curtain sign in lung ultrasound. J Med Ultrasound 2017. doi:10.1016/j.jmu.2017.04.005 